\title{
Propagation of ULF waves through the ionosphere: Analytic solutions for oblique magnetic fields
}

\author{
M. D. Sciffer and C. L. Waters \\ School of Mathematical and Physical Sciences and CRC for Satellite Systems, University of Newcastle, Callaghan, \\ New South Wales, Australia \\ Received 21 June 2001; revised 11 December 2001; accepted 18 March 2002; published 16 October 2002.
}

[1] The propagation of ultra low frequency (ULF; $1-100 \mathrm{mHz}$ ) waves from the magnetosphere to the ground is examined in the presence of oblique background magnetic fields. The problem is developed analytically for a thin sheet ionosphere, neutral atmosphere, and perfectly conducting ground. The cold plasma, ideal magnetohydrodynamic (MHD) Alfvén wave modes are assumed to propagate in the MHD medium above the ionosphere. A reflection and wave mode conversion coefficient matrix (RCM) is derived which describes mixing and conversion between shear Alfvén and fast mode energy when interacting with the ionosphere/atmosphere/ground system. The RCM is found to depend in a complicated way on the background magnetic field dip angle, the horizontal wave vector, and the conductivity of the ionosphere. For an oblique background magnetic field, $\vec{B}_{0}$ in the XZ plane, the perpendicular wave number, $k_{y}$, is shown to be a critical parameter that determines reflection and mode conversion characteristics. This study also highlights the need for spatial information of ULF wave energy in order to interpret experimental ULF wave data recorded at ground level in terms of magnetospheric processes. INDEX TERMS: 2736 Magnetospheric Physics: Magnetosphere/ ionosphere interactions; 2752 Magnetospheric Physics: MHD waves and instabilities; 2753 Magnetospheric Physics: Numerical modeling; KEYWORDS: ionosphere, magnetosphere, ULF waves

Citation: Sciffer, M. D., and C. L. Waters, Propagation of ULF waves through the ionosphere: Analytic solutions for oblique magnetic fields, J. Geophys. Res., 107(A10), 1297, doi:10.1029/2001JA000184, 2002.

\section{Introduction}

[2] Magnetohydrodynamic (MHD) waves propagate through the magnetosphere, interact with the ionosphere and may be detected using ground-based magnetometers. The ionosphere, atmosphere and ground system introduces important effects that alter the amplitude, polarization and spatial scale of these waves. ULF waves in the magnetosphere have wavelengths of order the size of the magnetosphere and carry information regarding magnetospheric dynamics. Remote sensing the magnetosphere using ground-based data is only possible if the effects of the ionosphere and atmosphere are included. Extracting relevant information from ground-based magnetometer arrays includes the evaluation of ULF wave polarization properties which are known to be altered by the ionosphere [Hughes, 1974; Hughes and Southwood, 1976a, 1976b; Glassmeier, 1984]. Furthermore, instruments that obtain in situ ionospheric data such as HF radars and ionospheric sounders also contain the effects of ULF wave energy [Poole et al., 1988; Menk et al., 1995]. Therefore, a thorough understanding of ULF wave propagation through the ionosphere to the ground is important for researchers and users of ULF and HF waves.

Copyright 2002 by the American Geophysical Union. 0148-0227/02/2001JA000184\$09.00
[3] Even though ULF wavelengths are much larger than the thickness of the ionosphere, the mathematical description of ULF wave propagation in the ionosphere and atmosphere system is nontrivial [e.g., Hughes, 1974]. The interaction of VLF waves with the ionosphere was investigated during the $1960 \mathrm{~s}$, in conjunction with radio wave propagation studies [e.g., Budden, 1985] and various numerical methods were developed to determine the wave properties through the changing ionosphere layers [e.g., Pitteway, 1965; Altman and Cory, 1969; Nygren, 1981, 1982]. The present study develops analytic solutions for ULF wave propagation in the magnetosphere, ionosphere and atmosphere environment in the presence of oblique background magnetic fields. Analytic solutions have previously been developed for the higher-frequency MHD waves (Pc1) [e.g., Manchester, 1968]. However, at these frequencies the waves can propagate along an Earth-ionosphere waveguide [e.g., Fraser, 1975]. For ULF waves, the vertical wavelengths are much larger and the problem becomes quite different.

[4] Dungey [1963] developed analytic solutions for the propagation of ULF energy through the ionosphere by dividing the problem into two parts. Any vector field may be expressed as the sum of toroidal (divergence free) and poloidal (curl free) fields. The fast and shear Alfvén MHD wave modes exist in the cold, magnetized plasma of the magnetosphere [e.g., Alfvén and Fälthammar, 1963]. The 
shear Alfvén mode has an associated field aligned current and curl free electric field while the fast mode is associated with the toroidal electric field. This method is still used, particularly for studying magnetosphere-ionosphere coupling [e.g., Yoshikawa and Itonaga, 2000]. Of particular interest was the 'ionospheric screening effect' associated with waves that carried field aligned current in the magnetosphere. Since no currents flow in the atmosphere, a discontinuity occurs in the ionosphere and by tracing $\nabla \times \vec{B}=\mu_{0} \vec{J}$ from the magnetosphere through to the atmosphere, the wave fields rotate $90^{\circ}$ for a horizontally uniform ionosphere. Nishida [1964] showed that this screening also applied to variations associated with storm sudden commencements. In the electrostatic limit where $\nabla \times \vec{e}=0$ in the ionosphere, Scholer [1970] showed that the reflection coefficient for a shear Alfvén wave is,

$$
A A_{\text {static }}=\frac{\Sigma_{a}-\Sigma_{p}}{\Sigma_{a}+\Sigma_{p}}
$$

where the Alfvén wave conductance is $\Sigma_{a}=\frac{1}{\mu_{0} V_{a}}$ and $\Sigma_{p}$ is the height integrated Pedersen conductivity.

[5] The first comprehensive, analytic treatment of the interaction of ULF waves with the ionosphere was reported in a series of papers by Hughes [1974] and Hughes and Southwood [1976a, 1976b]. Hughes [1974] developed analytic solutions in terms of the poloidal and toroidal fields of Dungey [1963]. The simplified system assumed horizontally uniform ionospheric conductivity, a perfect conducting ground, vertical background magnetic field and time and space harmonic variation fields. These papers showed the screening of the vertical current part of the solution and the associated $90^{\circ}$ polarization change of the wave through the ionosphere. Subsequent refinements for nonuniform ionosphere conductivity showed similar effects even though the polarization shift might be different from $90^{\circ}$ [Ellis and Southwood, 1983; Glassmeier, 1984].

[6] Tamao [1986] developed an analytic model in terms of field aligned currents (FACs) that considered the effect of an oblique background magnetic field and evaluated the contribution of the horizontal component of the FAC to the magnetic field observed on the ground. This was an electrostatic model where the inductive effect due to Faraday's law in the ionosphere was ignored as the frequencies were assumed to be low (in the Pc5 range). Furthermore, the model did not consider the effect of the ground on the solution.

[7] A number of subsequent analytic studies have provided reflection and transmission coefficients and Poynting flux details [e.g., Sorokin, 1986; Alperovich and Fedorov, 1992; Yoshikawa and Itonaga, 1996]. All these models assumed a vertical background magnetic field. From middle to low latitudes, the background magnetic field is oblique and analytic treatments to date have not treated this case. Prince and Bostick [1964] and Zhang and Cole [1995] have numerically solved the equatorial case. Signatures of MHD waves are observed on the ground at low latitudes and the ULF wave spectra contain both fast and shear Alfvén mode signatures [e.g., Waters et al., 1991, 1994; Menk et al., 1994]. Analytic treatments of the propagation of MHD waves across a plasma/vacuum interface show sensitivity of the results for background fields near vertical [e.g., Ullah

\section{Magnetosphere}

\section{Ideal MHD Region}

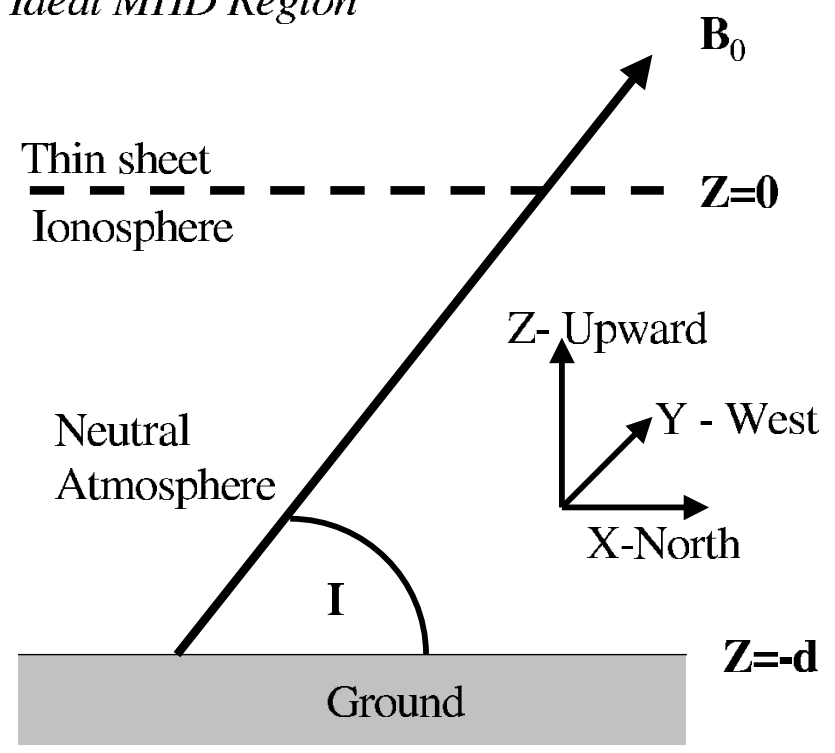

Figure 1. Geometry of the magnetosphere, ionosphere and atmosphere used for the ULF wave propagation model.

and Kahalas, 1963]. Therefore, we expect significant differences in the treatment for vertical background magnetic fields compared with lower latitude dip angles. In this paper we examine the propagation of ULF waves through a horizontally uniform, thin ionosphere where the background magnetic field is oblique, including the effects of the neutral atmosphere and reflection from the ground. This is achieved by developing analytical expressions using a wave rather than a FAC formulation and examining the reflection and transmission properties. These are developed for comparison with numerical solutions and two dimensional simulations presently under development.

\section{ULF Wave Model}

[8] The ionosphere is approximated by a thin sheet in the XY plane at $z=0$ as shown in Figure 1. A 'thin' ionosphere means that the thickness of the region is much less than the wavelength in that direction, i.e. $H_{z}^{I} \ll \lambda_{z}$, where $H_{z}^{I}$ is the thickness of the ionosphere in the $\mathrm{Z}$ direction. Therefore, the vertical current in the ionospheric sheet is set to zero. The properties of the ionosphere are described by height integrated conductivities. The magnetosphere is described by ideal MHD, where the field aligned electric field is zero. In the atmosphere we assume $j^{a t m}=0$ and the ground is modeled as a perfect conductor.

\subsection{Boundary Conditions}

[9] Analytic solutions for ULF waves in an ideal, MHD plasma are well known [e.g., Stix, 1962]. In the cold plasma that populates the magnetosphere two MHD wave modes exist. The fast (or compressional) mode is isotropic in nature and the shear Alfvén (or torsional) mode carries energy along the background magnetic field [Priest, 1982; Cross, 1988]. The wave modes in the neutral atmosphere are also well known and are described by the solution of the 
linearized Faraday and Ampere laws. The possible wave modes are the transverse electric and magnetic (TEM) mode, the transverse magnetic (TM) mode and the transverse electric (TE) mode [e.g., Cheng, 1983]. Given that analytic solutions in both the magnetosphere and atmosphere are known, the problem is to match these solutions across the ionospheric current sheet.

[10] The boundary conditions for electromagnetic phenomena are [Cheng, 1983] (1) tangential components of $\vec{e}$ are continuous across the boundary, (2) normal components of $\vec{b}$ are continuous across the boundary (i.e. $b_{z}^{\text {mag }}=b_{z}^{\text {atm }}$ ), (3) tangential components of $\vec{b}$ are discontinuous across the boundary. The discontinuity is described by

$$
\begin{aligned}
& \vec{n}_{z} \times\left(\vec{b}^{\text {mag }}-\vec{b}^{a t m}\right)=(0,0,1) \times\left(\underset{z \rightarrow 0^{+}}{\lim } \vec{b}_{\perp}^{\text {mag }}-\underset{z \rightarrow 0^{-}}{\lim } \vec{b}_{\perp}^{a t m}\right) \\
& =\mu_{0} \vec{J}_{s}=\mu_{0}\left(j_{x}, j_{y}, 0\right)
\end{aligned}
$$

where $\vec{J}_{S}$ is the current density in the sheet, $\vec{b}_{\perp}$ is the magnetic field perpendicular to $\vec{n}_{z}$ and (4) the normal components of $\vec{e}$ are discontinuous across the boundary when a surface charge exists. For an incident ULF wave, the incident, reflected and transmitted components all meet at the boundary where condition 1 implies that the incident, reflected and transmitted waves must have the same frequency and the same wavelength parallel to the boundary [Cross, 1988].

\subsection{Currents in the Ionosphere}

[11] The background magnetic field, $\vec{B}_{0}$, is confined to the $\mathrm{XZ}$ plane as shown in Figure 1 so that

$$
\overrightarrow{B_{0}}=B_{0}[\cos (I), 0, \sin (I)]
$$

The current density in the ionosphere is related to the electric field by the conductivity tensor. The oblique orientation of the background magnetic field complicates the elements of the conductivity tensor compared with vertical or horizontal cases. Assuming that the ionosphere is horizontally uniform, the conductivity tensor for an oblique background magnetic field is

$\bar{\Sigma}=\left[\begin{array}{ccc}\Sigma_{d} \cos ^{2}(I)+\Sigma_{p} \sin ^{2}(I) & \Sigma_{h} \sin (I) & \left(\Sigma_{d}-\Sigma_{p}\right) \sin (I) \cos (I) \\ -\Sigma_{h} \sin (I) & \Sigma_{p} & \Sigma_{h} \cos (I) \\ \left(\Sigma_{d}-\Sigma_{p}\right) \sin (I) \cos (I) & -\Sigma_{h} \cos (I) & \Sigma_{d} \sin ^{2}(I)+\Sigma_{p} \cos ^{2}(I)\end{array}\right]$

where $\Sigma_{d}, \Sigma_{p}$ and $\Sigma_{h}$ are the height integrated direct, Pedersen and Hall conductivities respectively.

[12] The first-order perturbation currents in the ionosphere are

$$
\begin{aligned}
& j_{x}^{I}=\sigma_{11} e_{x}^{I}+\sigma_{12} e_{y}^{I}+\sigma_{13} e_{z}^{I} \\
& j_{y}^{I}=\sigma_{21} e_{x}^{I}+\sigma_{22} e_{y}^{I}+\sigma_{23} e_{z}^{I} \\
& j_{z}^{I}=\sigma_{31} e_{x}^{I}+\sigma_{32} e_{y}^{I}+\sigma_{33} e_{z}^{I}=0
\end{aligned}
$$

where $\sigma_{i j}$ is the $i$ th row and $j$ th column element of the height integrated conductivity tensor, $\bar{\Sigma}$, in (4). The first-order perturbed electric fields arising from ULF wave energy in the ionosphere are $e_{x}^{I}, e_{y}^{I}$ and $e_{z}^{I}$. Equation (7) sets $j_{z}^{I}$ to zero since the current sheet is parallel to the ground. From (7) the vertical electric field in the ionosphere is

$$
e_{z}^{I}=-\frac{\sigma_{31} e_{x}^{I}+\sigma_{32} e_{y}^{I}}{\sigma_{33}}
$$

\subsection{Boundary Equations}

[13] The boundary conditions may be expressed in terms of the electric fields in the atmosphere and magnetosphere. Define a symbol $\Delta$ such that

$$
\Delta F=\lim _{z \rightarrow 0^{+}} F^{m a g}-\underset{z \rightarrow 0^{-}}{\lim } F^{a t m}
$$

where $F^{\text {mag }}$ and $F^{\text {atm }}$ indicate magnetospheric and atmospheric variables respectively. The boundary condition given by (2) becomes,

$$
\vec{n}_{z} \times(\Delta \vec{b})=\left(-\Delta b_{y}, \Delta b_{x}, 0\right)=\mu_{0}\left(j_{x}^{I}, j_{y}^{I}, 0\right)
$$

[14] Assuming a temporal dependence of $e^{-i \omega t}$, Faraday's law is

$$
i \omega \vec{b}=\nabla \times \vec{e} .
$$

The importance of the inductive effect from (11) is a recent development in magnetosphere/ionosphere coupling [e.g., Yoshikawa and Itonaga, 1996] and we also include this term. The contribution from $i \omega \vec{b}$ on the solution depends on the frequency of the waves. For Pc5 $(1-5 \mathrm{mHz})$ the influence of (11) is minimal. However, for frequencies in the Pc3 (20-100 $\mathrm{mHz}$ ) range, routinely observed at mid to low latitudes, the inductive effect becomes important.

[15] The transverse magnetic field perturbations from (11) may be substituted into the boundary condition given by (10) and using (5) to (9),

$$
\begin{aligned}
& \mu_{0}\left(\sigma_{11}+\frac{\sigma_{31} \sigma_{13}}{\sigma_{33}}\right) e_{x}^{I}+\mu_{0}\left(\sigma_{12}+\frac{\sigma_{32} \sigma_{13}}{\sigma_{33}}\right) e_{y}^{I} \\
& -\frac{i}{\omega}\left(\lim _{z \rightarrow 0^{+}}\left(\frac{\partial e_{x}^{m}}{\partial z}-\frac{\partial e_{z}^{m}}{\partial x}\right)-\lim _{z \rightarrow 0^{-}}\left(\frac{\partial e_{x}^{a}}{\partial z}-\frac{\partial e_{z}^{a}}{\partial x}\right)\right)=0
\end{aligned}
$$

$$
\begin{aligned}
& \mu_{0}\left(\sigma_{21}+\frac{\sigma_{31} \sigma_{23}}{\sigma_{33}}\right) e_{x}^{I}+\mu_{0}\left(\sigma_{22}+\frac{\sigma_{32} \sigma_{23}}{\sigma_{33}}\right) e_{y}^{I} \\
& -\frac{i}{\omega}\left(\lim _{z \rightarrow 0^{+}}\left(\frac{\partial e_{z}^{m}}{\partial y}-\frac{\partial e_{y}^{m}}{\partial z}\right)-\lim _{z \rightarrow 0^{-}}\left(\frac{\partial e_{z}^{a}}{\partial y}-\frac{\partial e_{y}^{a}}{\partial z}\right)\right)=0
\end{aligned}
$$

Equations (12) and (13) specify the relationship between the electric fields near the thin sheet ionosphere boundary and they involve the electric fields in the magnetosphere and atmosphere. The electric fields in the magnetosphere for oblique background magnetic fields are developed next, followed by expressions for the electric field in the atmosphere.

\subsection{Wave Properties in the Magnetosphere}

[16] For ideal MHD conditions, any electric field must be perpendicular to $\vec{B}_{0}$ (i.e. $e_{\|}=0$ ). When $\vec{B}_{0}$ is oblique and in the $\mathrm{XZ}$ plane, 


$$
e_{\|}=e_{x} \cos (I)+e_{z} \sin (I)=0
$$

and so

$$
e_{z}=-e_{x} \cot (I)
$$

The two cold plasma, ideal MHD, wave modes may be identified by the relationship between the electric fields and wave numbers. Assuming an $e^{i\left(k_{x} x+k_{y} y\right)}$ horizontal wave structure, the fast (or compressional) mode has

$$
\nabla \cdot \vec{e}=0 \quad(\nabla \times \vec{e})_{\|} \neq 0
$$

and the dispersion relation is [eg. Cross, 1988]

$$
\frac{\omega^{2}}{V_{a}^{2}}=k_{x}^{2}+k_{y}^{2}+k_{z, f}^{2}
$$

The vertical wave number for a fast mode is,

$$
k_{z, f}= \pm \sqrt{\frac{\omega^{2}}{V_{a}^{2}}-k_{x}^{2}-k_{y}^{2}}
$$

From (15), (16) and (18) the electric field associated with the fast mode is,

$$
e^{f}=\beta\left[-k_{y} \sin (I), k_{x} \sin (I)-k_{z, f} \cos (I), k_{y} \cos (I)\right]
$$

where $\beta$ is a complex constant and $I$ is the dip angle of the background magnetic field.

[17] For a shear Alfvén mode

$$
\nabla \cdot \vec{e} \neq 0 \quad(\nabla \times \vec{e})_{\|}=0
$$

and the dispersion relation is

$$
\frac{\omega^{2}}{V_{a}^{2}}=\left[k_{x} \cos (I)+k_{z, a} \sin (I)\right]^{2}
$$

Therefore, the vertical wave number for the shear Alfvén mode is

$$
k_{z, a}=\frac{ \pm \frac{\omega}{V_{a}}-k_{x} \cos (I)}{\sin (I)}
$$

For oblique $\vec{B}_{0}$, (22) shows that the shear Alfvén mode has two different wave numbers in the vertical direction. From (20), (15) and (21) the electric field associated with the shear Alfvén mode is

$$
\begin{aligned}
e^{a}= & \alpha\left[\left(k_{x} \sin (I)-k_{z, a} \cos (I)\right) \sin (I), k_{y},-\left(k_{x} \sin (I)\right.\right. \\
& \left.\left.-k_{z, a} \cos (I)\right) \cos (I)\right]
\end{aligned}
$$

where $\alpha$ is a complex constant.

[18] The total electric field in the magnetosphere is a superposition of the incident and reflected waves. There- fore, from (19) and (23) the total electric field components are

$$
\left(\begin{array}{c}
e_{x}^{m a g} \\
e_{y}^{m a g} \\
e_{z}^{m a g}
\end{array}\right)=\alpha^{r} \vec{P}_{a}^{r}+\alpha^{i} \vec{P}_{a}^{i}+\beta^{r} \vec{P}_{f}^{r}+\beta^{i} \vec{P}_{f}^{i}
$$

where $a$ denotes the shear Alfvén mode, $f$ is the fast mode, $i$ denotes incident and $r$ is for the reflected wave. Therefore, $\vec{P}_{m}^{d}$ denotes the unit vector in the direction of the electric field of the appropriate MHD wave mode. The $\alpha$ and $\beta$ are amplitude factors from (19) and (23). This superposition of the magnetospheric electric field allows the wave fields and their derivatives to be expressed in terms of the composition of MHD wave modes present in the magnetosphere. The polarization of each wave mode is contained in the $\vec{P}_{m}^{d}$.

\subsection{Wave Properties in the Atmosphere}

[19] No currents flow in the neutral atmosphere and Maxwell's equations give,

$$
\mu_{0} \epsilon_{0} \frac{\partial^{2} \vec{e}}{\partial t^{2}}+\nabla^{2} \vec{e}=0
$$

so that

$$
k_{z}^{a t m}=\sqrt{\frac{\omega^{2}}{c^{2}}-\left(k_{x}^{2}+k_{y}^{2}\right)} .
$$

The boundary conditions at the ionosphere/atmosphere interface specify continuity of the horizontal electric fields at $z=0$ so that

$$
e_{x}^{a t m}(0)=e_{x}^{i o n}(0)=e_{x}^{m a g}(0) \quad \text { and } \quad e_{y}^{a t m}(0)=e_{y}^{i o n}(0)=e_{y}^{m a g}(0) .
$$

At the neutral atmosphere/ground interface, the ground is a perfect conductor at $z=-d$ so that

$$
e_{x}^{a t m}(-d)=e_{y}^{a t m}(-d)=0 .
$$

Assuming that $k_{x}^{2}+k_{y}^{2}>\frac{\omega^{2}}{c^{2}}$, the solution in the atmosphere is given by

$$
\begin{aligned}
& e_{x}^{a t m}=\gamma_{x} \frac{\sinh \left(i k_{z}^{a t m}(z+d)\right)}{\sinh \left(i k_{z}^{a t m}(d)\right)} e^{i\left(k_{x} x+k_{y} y\right)} \\
& e_{y}^{a t m}=\gamma_{y} \frac{\sinh \left(i k_{z}^{a t m}(z+d)\right)}{\sinh \left(i k_{z}^{a t m}(d)\right)} e^{i\left(k_{x} x+k_{y} y\right)} \\
& e_{z}^{\text {atm }}=\gamma_{z} \frac{\cosh \left(i k_{z}^{a t m}(z+d)\right)}{\sinh \left(i k_{z}^{\text {atm }}(d)\right)} e^{i\left(k_{x} x+k_{y} y\right)}
\end{aligned}
$$

where $\gamma_{i}$ is a complex constant and represents the amplitude of the component of the wave just below the current sheet (i.e. at $z=0^{-}$). Given the continuity of horizontal electric fields through the ionosphere, we can express the amplitude 
of the atmospheric electric fields just below the current sheet as

$$
\begin{gathered}
\gamma_{x}=e_{x}^{m a g}(0)=\alpha^{i} P_{x, a}^{i}+\alpha^{r} P_{x, a}^{r}+\beta^{i} P_{x, f}^{i}+\beta^{r} P_{x, f}^{r} \\
\gamma_{y}=e_{y}^{m a g}(0)=\alpha^{i} P_{y, a}^{i}+\alpha^{r} P_{y, a}^{r}+\beta^{i} P_{y, f}^{i}+\beta^{r} P_{y, f}^{r} \\
\gamma_{z}=\frac{-k_{x} \gamma_{x}-k_{y} \gamma_{y}}{k_{z}^{a t m}}
\end{gathered}
$$

where $\gamma_{z}$ has been calculated from $\nabla \cdot \vec{e}^{a t m}=0$ and the boundary conditions given in (27).

\subsection{Reflection and Wave Mode Conversion Coefficient Matrix}

[20] Substituting (29) to (31) and (24) for $z=0$ into (12) and (13) gives

$$
\Theta^{r}\left[\begin{array}{l}
\alpha^{r} \\
\beta^{r}
\end{array}\right]=\Theta^{i}\left[\begin{array}{l}
\alpha^{i} \\
\beta^{i}
\end{array}\right]
$$

where $\Theta^{i}$ and $\Theta^{r}$ are 2 by 2 matrices of the form

$$
\Theta=\left[\begin{array}{ll}
a & b \\
c & d
\end{array}\right]
$$

where

$$
\begin{aligned}
a= & S_{11} P_{x, a}+S_{12} P_{y, a}-\frac{P_{z, a} k_{x}}{\omega}+\frac{P_{x, a} k_{z, a}}{\omega} \\
& +\frac{k_{x} \xi_{z, a}}{\omega} \tanh \left(-i k_{z}^{a t m} d\right)-\frac{k_{z}^{a t m} P_{x, a}}{\omega} \tanh \left(-i k_{z}^{a t m} d\right) \\
b= & S_{11} P_{x, f}+S_{12} P_{y, f}-\frac{P_{z, f} k_{x}}{\omega}+\frac{P_{x, f} k_{z, f}}{\omega} \\
& +\frac{k_{x} \xi_{z, f}}{\omega} \tanh \left(-i k_{z}^{a t m} d\right)-\frac{k_{z}^{a t m} P_{x, f}}{\omega} \tanh \left(-i k_{z}^{\text {atm }} d\right) \\
c= & S_{21} P_{x, a}+S_{22} P_{y, a}-\frac{P_{z, a} k_{y}}{\omega}+\frac{P_{y, a} k_{z, a}}{\omega} \\
& +\frac{k_{y} \xi_{z, a}}{\omega} \tanh \left(-i k_{z}^{a t m} d\right)-\frac{k_{z}^{a t m} P_{y, a}}{\omega} \tanh \left(-i k_{z}^{\text {atm }} d\right) \\
d= & S_{21} P_{x, f}+S_{22} P_{y, f}-\frac{P_{z, f} k_{y}}{\omega}+\frac{P_{y, f} k_{z, f}}{\omega} \\
& +\frac{k_{y} \xi_{z, f}}{\omega} \tanh \left(-i k_{z}^{a t m} d\right)-\frac{k_{z}^{a t m} P_{y, f}}{\omega} \tanh \left(-i k_{z}^{a t m} d\right) .
\end{aligned}
$$

$P_{c, m}$ indicates the MHD mode and the spatial component of the unit vector, $\xi_{z, a}=-\left(k_{x} P_{x, a}+k_{y} P_{y, a}\right) / k_{z}^{\text {atm }}$ and $\xi_{z, f}=$ $-\left(k_{x} P_{x, f}+k_{y} P_{y, f}\right) / k_{z}^{\text {atm }}$. The $\Theta^{i}$ and $\Theta^{r}$ depend upon whether the wave is incident or reflected from the ionosphere. Vertical wave numbers for the fast and shear Alfvén modes and the vertical wave number in the atmosphere are $k_{z, f}, k_{z, a}$ and $k_{z}^{a t m}$ respectively. The $S_{i, j}$ represent terms involving the conductivities of the ionosphere for an oblique $\vec{B}_{0}$ where

$$
\begin{aligned}
& S_{11}=\mu_{0}\left(\sigma_{11}+\frac{\sigma_{31} \sigma_{13}}{\sigma_{33}}\right) \\
& S_{12}=\mu_{0}\left(\sigma_{12}+\frac{\sigma_{32} \sigma_{13}}{\sigma_{33}}\right) \\
& S_{21}=\mu_{0}\left(\sigma_{21}+\frac{\sigma_{31} \sigma_{23}}{\sigma_{33}}\right) \\
& S_{22}=\mu_{0}\left(\sigma_{22}+\frac{\sigma_{32} \sigma_{23}}{\sigma_{33}}\right) .
\end{aligned}
$$

From (35), the amplitudes of the reflected MHD modes are

$$
\left[\begin{array}{l}
\alpha^{r} \\
\beta^{r}
\end{array}\right]=\left(\Theta^{r}\right)^{-1} \Theta^{i}\left[\begin{array}{l}
\alpha^{i} \\
\beta^{i}
\end{array}\right]=\left[\begin{array}{ll}
\Gamma_{11} & \Gamma_{12} \\
\Gamma_{21} & \Gamma_{22}
\end{array}\right]\left[\begin{array}{c}
\alpha^{i} \\
\beta^{i}
\end{array}\right]
$$

In terms of (37)-(40),

$$
\left[\begin{array}{ll}
\Gamma_{11} & \Gamma_{12} \\
\Gamma_{21} & \Gamma_{22}
\end{array}\right]=\frac{1}{a^{r} d^{r}-b^{r} c^{r}}\left[\begin{array}{cc}
a^{i} d^{r}-c^{i} b^{r} & b^{i} d^{r}-d^{i} b^{r} \\
-a^{i} c^{r}+c^{i} a^{r} & -b^{i} c^{r}+d^{i} a^{r}
\end{array}\right]
$$

where the subscripts $r$ and $i$ indicate where the reflected or incident forms of $a, b, c$ and $d$ are to be used.

[21] The resultant 2 by 2 RCM matrix contains the elements, $\Gamma_{i j}$, which describe the reflection and mode conversion properties of ULF waves for the combined ionosphere/atmosphere/ground system. The elements of the RCM are defined by (37)-(40) and (45). The contribution of the incident shear Alfvén mode in the reflected shear Alfvén mode is determined by $\Gamma_{11}$, the contribution of the incident fast mode in the reflected shear Alfvén mode is determined by $\Gamma_{12}$ and so on. The $\Gamma_{i j}$ shall be denoted by $\mathrm{AA}, \mathrm{FA}, \mathrm{AF}$ and FF to be consistent with previous studies and to indicate more readily the combination of modes involved. They all depend on dip angle, the three ionospheric conductivities, ULF disturbance wave numbers, wave frequency and the height of the ionospheric current sheet above the ground. The RCM provides important information regarding ULF mode conversion and reflection characteristics. However, measured quantities are the electric and/or magnetic field perturbations which must be constructed using both (24) and (45).

\section{Properties of the Reflection and Wave Mode Conversion Coefficient Matrix}

[22] The spatial structure of ULF waves in the presence of oblique $\vec{B}_{0}$ has important effects on the reflection and mode conversion properties. The wave structure in the horizontal plane may involve complex wave numbers. However, in the following results both horizontal wave numbers have been constrained to real numbers. Since $\vec{B}_{0}$ lies in the XZ plane, the projection of the horizontal wave number onto an oblique $\vec{B}_{0}$ has a significant effect on the RCM. For non vertical $\vec{B}_{0}$ there is a projection of the horizontal wave number in the direction of the background field only if $k_{x} \neq 0$. The vertical wave number for the shear Alfvén mode, $k_{z, a}$, depends on the choice of $k_{x}$, while for the fast 
mode, the vertical wave number, $k_{z, f}$ depends on both $k_{x}$ and $k_{y}$, according to the appropriate dispersion relations given by (17) and (21). In the following results the wave numbers are chosen so that both the fast and shear Alfvén modes propagate.

\subsection{Variations in Horizontal Wave Number}

\subsection{1. $k_{x} \neq 0, k_{y}=0$}

[23] The modulus, real part and phase of the four elements of the RCM, as a function of magnetic field dip angle for fixed horizontal wave numbers, conductivities and frequency are shown in Figure 2. The horizontal axis represents Northern Hemisphere (-ve) through to Southern Hemisphere $(+v e)$ dip angles. The magnitude of AA for $k_{x}=\frac{\omega}{2 V_{a}}$ and $k_{y}=0$ shows little variation with dip angle and is close to unity. Similar trends are seen in the modulus and real part of FF. The contribution of the incident shear Alfvén mode to reflected fast mode, AF, is small but has a maximum for vertical $\vec{B}_{0}$. The contribution of the incident fast mode to reflected shear Alfvén mode, FA, is also small and this is the case whenever $k_{y}=0$. All coefficients show a $180^{\circ}$ phase change around dip angles of $\pm 60^{\circ}$. If the magnitude of $k_{x}$ is increased (decreased) then these phase changes occur at more equatorial (poleward) dip angles. FF is symmetric about the equator in all cases where $k_{y}=0$.

[24] In order to appreciate the structure of the total wave fields in the MHD medium, consider the case of an incident shear Alfvén wave only and $\mathrm{AF}=0$. The absolute value of AA indicates amplitude change on reflection. Combining this with the phase of AA determines the polarization of the total wave field. When more than one reflection coefficient is nonzero, a polarization state may be defined for each combination of Alfvén and fast mode, incident and reflected. The total wave field in the MHD medium is the superposition of the product of the wave amplitudes $(\alpha, \beta)$ with their respective polarization states, $P_{m}^{d}$, according to (24).

3.1.2. $k_{x}=0, k_{y} \neq 0$

[25] Setting $k_{y} \neq 0$ has a pronounced effect on the RCM as shown in Figure 3. Both AA and FF exhibit maxima when $\vec{B}_{0}$ is near vertical and horizontal (equatorial). Minima appear near $I= \pm 60^{\circ}$ and these move to other dip angles according to $k_{y}$. As $k_{y}$ increases (decreases), the minima occur at more equatorial (poleward) dip angles. These minima correspond with transitions from $180^{\circ}$ to $0^{\circ}$ phase reflection. The transition from $180^{\circ}$ to $0^{\circ}$ reflection for $\mathrm{FF}$ is smoother compared with Figure 2.

[26] Both AF and FA are very small when $\vec{B}_{0}$ is vertical. The maxima in $|\mathrm{AF}|$ occur where $|\mathrm{AA}|$ has local minima and $|\mathrm{AF}|$ decreases to zero for an equatorial magnetic field. The magnitude of FA remains close to unity for low and midlatitude dip angles although $|\mathrm{FA}|$ decreases in this region as $k_{y}$ decreases. This indicates that the contribution of the incident fast mode converted to reflected shear Alfvén mode (FA) is greater than AF for low and midlatitudes but this has a $k_{y}$ dependence. The AF coefficient is predominantly real so the phase is zero for all dip angles. In all cases when $k_{x}=0$ the RCM coefficients are symmetric about the equator.

\subsection{3. $k_{x} \neq 0, k_{y} \neq 0$}

[27] The dependence of the elements of the RCM on magnetic field dip angle for nonzero $k_{x}$ and $k_{y}$ is shown in
Figure 4. Comparing Figures 2 through 4 shows that the wave number perpendicular to the plane of the background magnetic field, $k_{y}$, is the dominant influence in determining the dependence of the RCM elements on dip angle. While FA and AF show an asymmetry about the equator, $|\mathrm{AA}|$ and $|\mathrm{FF}|$ are symmetric. A non zero $k_{x}$ and $k_{y}$ gives an asymmetry about the equator in FA and $\mathrm{AF}$ as a function of dip angle. In Figure 4 the dip angles where $|\mathrm{FF}|$ and $|\mathrm{AA}|$ show minima depend on both $k_{x}$ and $k_{y}$ and are now shifted from $\pm 60^{\circ}$.

\subsection{Variations in Conductivity}

[28] Variations in the height integrated conductivities were found to have quite complicated effects on the elements of the RCM for all dip angles. As shown in the previous section, the elements of the RCM also depend on the wave numbers. In order to highlight the effect of conductivity changes, representative horizontal wave numbers of $k_{x}=k_{y}=\frac{\omega}{2 V_{t}}$ have been chosen. In this case, there is a component of the wave number perpendicular to the plane in which $\vec{B}_{0}$ varies (non zero $k_{y}$ ). Having fixed the wave numbers, we may vary the height integrated Hall and/or Pedersen conductivities and plot the RCM elements as a function of dip angle. Typical day time, height integrated conductivities are about $\Sigma_{p, h}=$ $10 \mathrm{~S}$. In order to show the marked changes of the RCM with conductivity, values for both $\Sigma_{p, h}=10$ and $1 \mathrm{~S}$ are included.

\subsection{1. $\Sigma_{p}=1$ and $10 \mathrm{~S}$, Vary $\Sigma_{h}$}

[29] The variation of the elements of the RCM as the height integrated Hall conductivity was varied from 0.1 to $100 \mathrm{~S}$ is shown in Figure 5. The left-hand panels are for $\Sigma_{p}=1 \mathrm{~S}$ while the right-hand panels are for $\Sigma_{p}=10 \mathrm{~S}$. As $\Sigma_{p}$ increases, the RCM becomes less dependent on changes in $\Sigma_{h}$. The variation in $|\mathrm{AA}|$ with conductivity for vertical dip angles agrees with those shown by Yoshikawa et al. [1999] where the minimum value of $|\mathrm{AA}|$ depends on $\Sigma_{p}$. For the larger $\Sigma_{p}$ (right-hand panels), any slice at constant $\Sigma_{h}$ would correspond with Figure 4. Minima in $|\mathrm{AA}|$ correspond with maxima in $|\mathrm{AF}|$ around $\pm 50^{\circ}$ dip angle. The asymmetry in $|\mathrm{AF}|$ is due to the non zero $k_{x}$ in the shear Alfvén mode dispersion relation giving asymmetric $k_{z}, a$. If $k_{x}$ changes sign, then the magnitude and phase variations flip around the $I=0$ line. If $k_{x}=0$ then the results are symmetric around the equator. Variations in the wave numbers change the dip angles where maxima and minima in the RCM occur.

3.2.2. $\Sigma_{h}=1$ and $10 \mathrm{~S}$, Vary $\Sigma_{p}$

[30] The reflection coefficient in the electrostatic limit, of the shear Alfvén mode for vertical $\vec{B}_{0}$ involves the Pedersen conductivity according to (1). This can be compared with our AA which includes both inductive and oblique $\vec{B}_{0}$ effects. The variation of the elements of the RCM as the height integrated Pedersen conductivity was varied from 0.1 to $100 \mathrm{~S}$ is shown in Figure 6. The reflection coefficient in (1) is zero when $\Sigma_{a}=\Sigma_{p}$ which for the values chosen is 0.8 . For vertical $\vec{B}_{0}$ and small $\Sigma_{h}$ (left-hand panels), $|\mathrm{AA}|$ shows a minimum close to 0.8 , in agreement with the electrostatic limit. For the larger $\Sigma_{h}$ (right-hand panels), the inductive effect modifies the RCM so that the vertical $\vec{B}_{0},|\mathrm{AA}|$ minimum is non zero and shifted to $\Sigma_{p}=2.1$. The asymmetry in $|\mathrm{AF}|$ and $|\mathrm{FA}|$ about the equator highlights 
$|A A|$

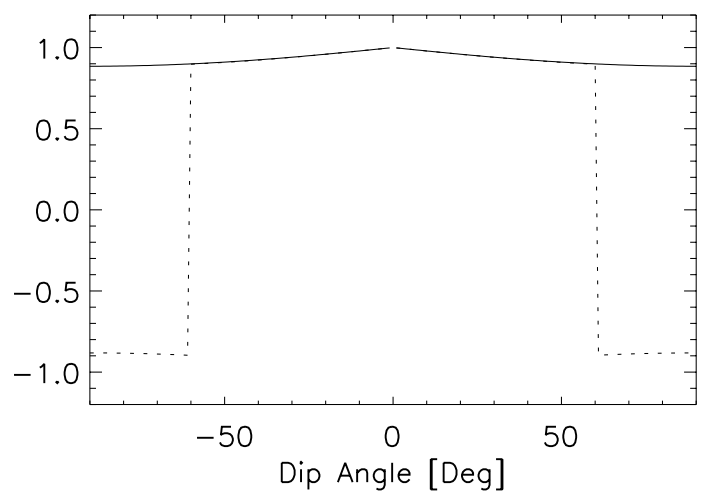

$|A F|$

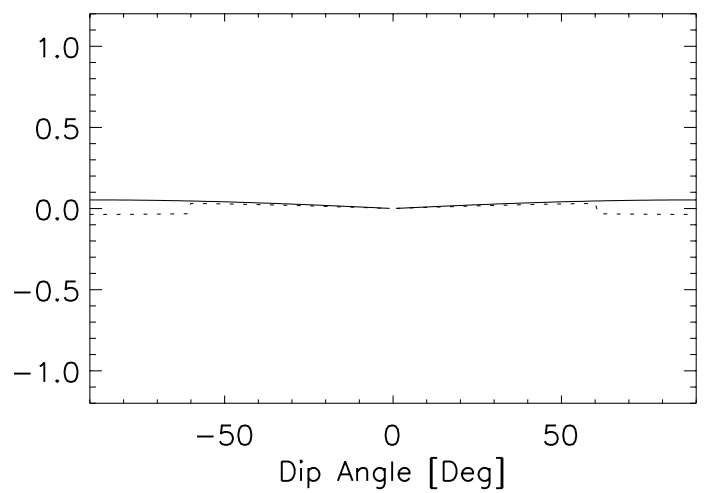

$|F A|$

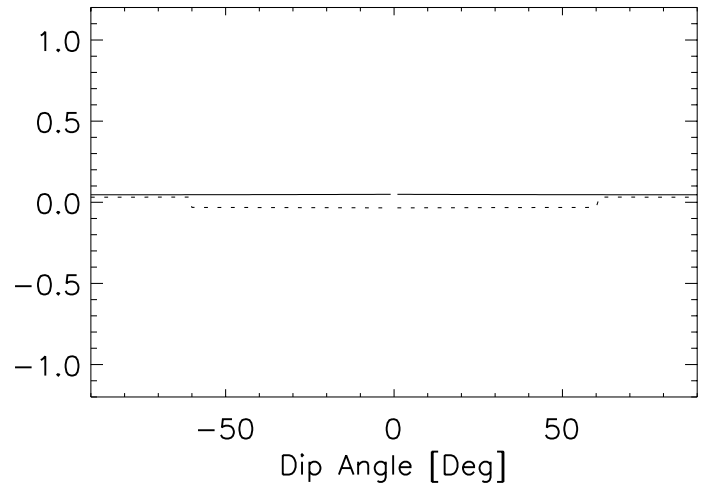

$|F F|$

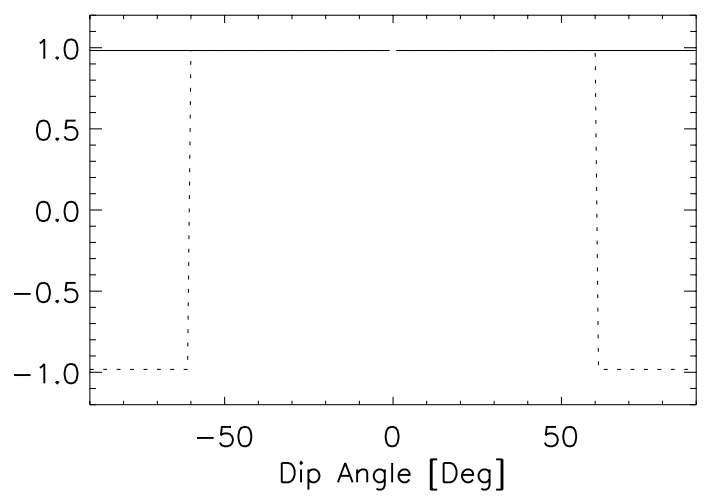

AA Phase
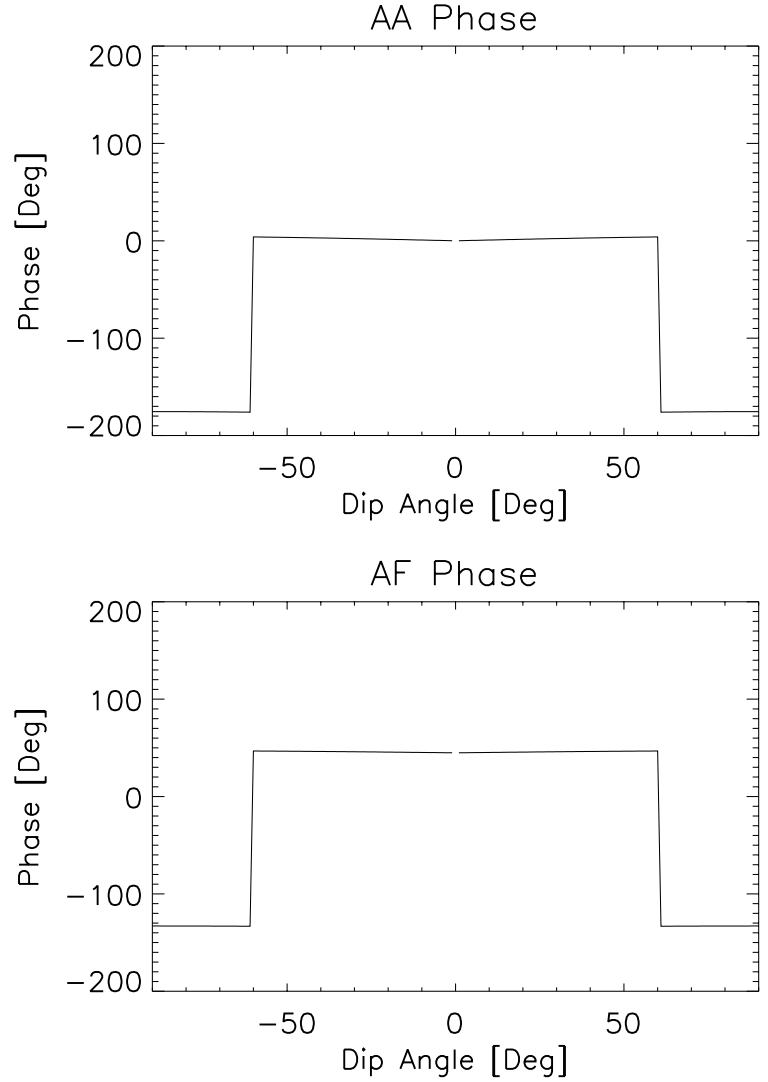

FA Phase

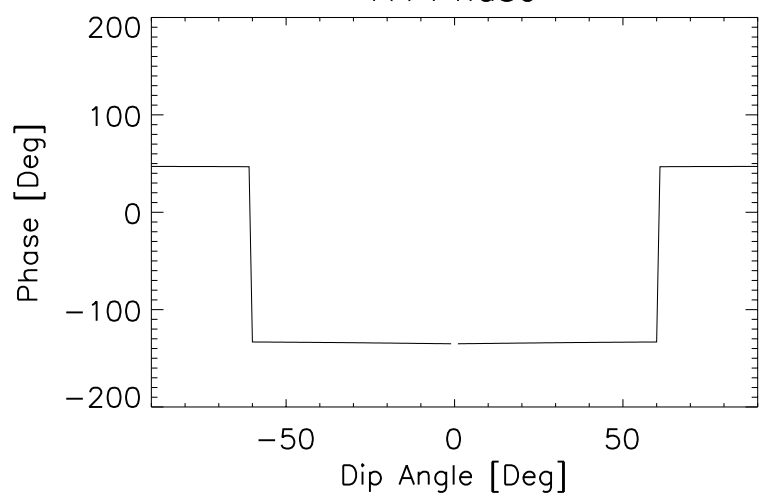

FF Phase

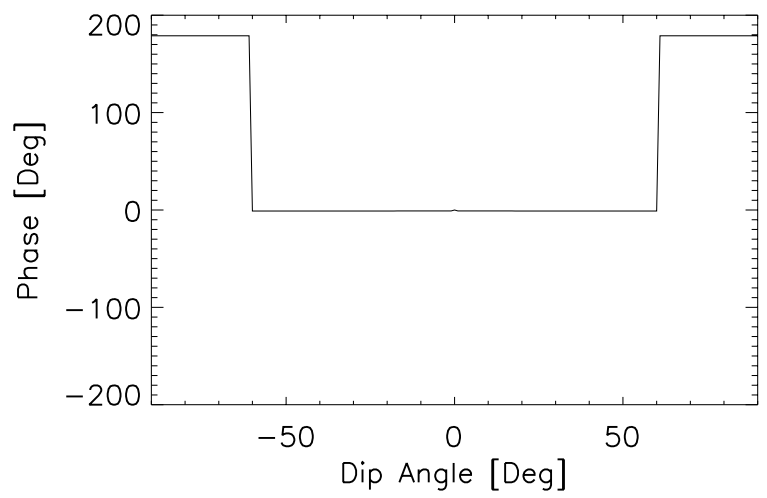

Figure 2. Reflection coefficient matrix elements for $k_{x}=\frac{\omega}{2 V}, k_{y}=0$, ionosphere height, $\mathrm{d}=1.25 \times 10^{5}$ $\mathrm{m}$, Alfvén speed, $V_{a}=2 \pi d$, direct height integrated conductivity, $\Sigma_{d}=1,000,000 \mathrm{~S}$, Pedersen height integrated conductivity, $\Sigma_{p}=10 \mathrm{~S}$, Hall height integrated conductivity, $\Sigma_{h}=20 \mathrm{~S}$ and ULF wave frequency, $\mathrm{f}=20 \mathrm{mHz}$. The electrostatic value from (1) for AA in this case is $A A_{\text {static }}=-0.85$. 

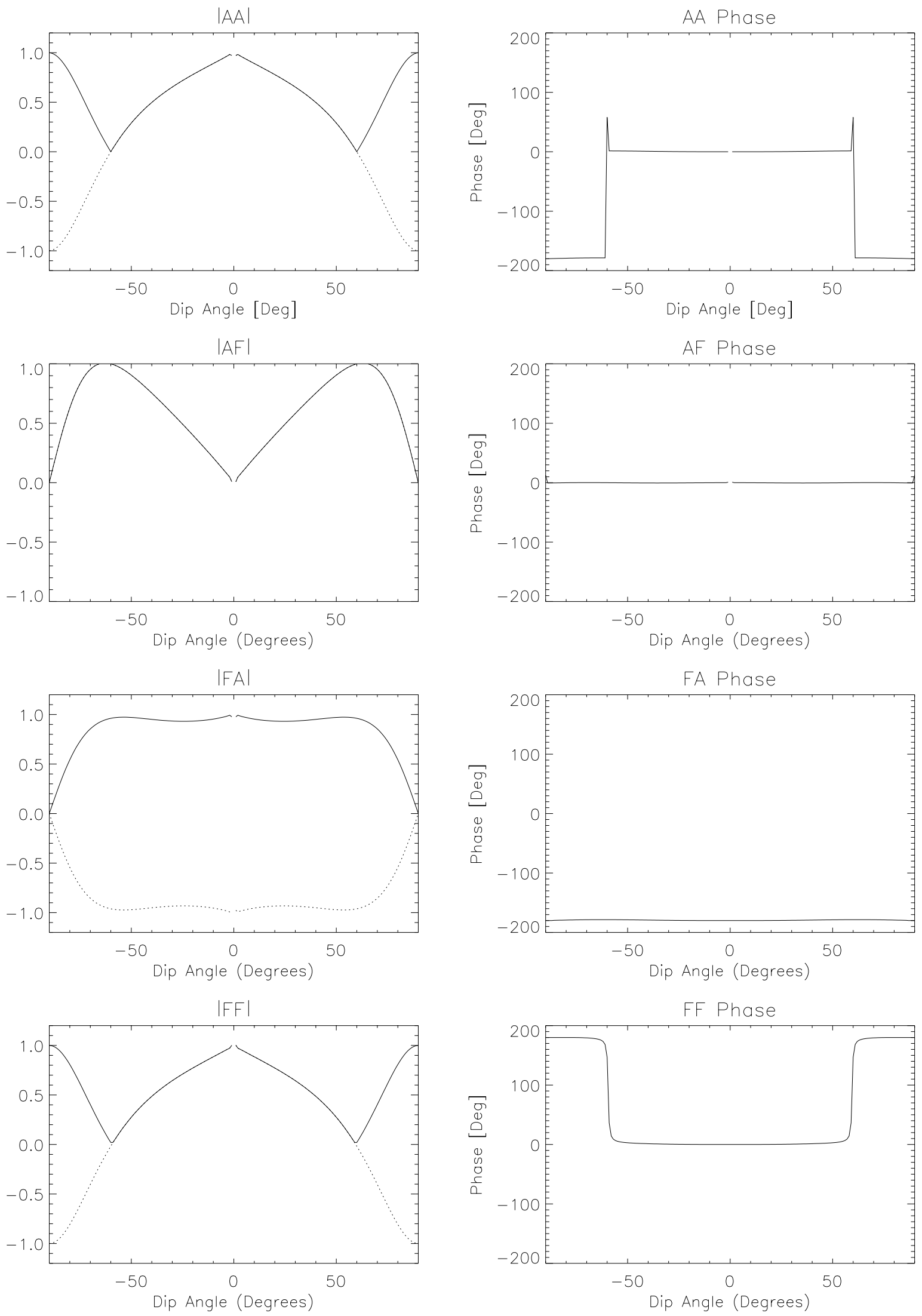

Figure 3. Reflection coefficient matrix elements for $k_{x}=0, k_{y}=\frac{\omega}{2 V}$, ionosphere height, $\mathrm{d}=1.25 \times 10^{5} \mathrm{~m}$, Alfvén speed, $V_{a}=2 \pi d$, direct height integrated conductivity, $\Sigma_{d}=1,000,000 \mathrm{~S}$, Pedersen height integrated conductivity, $\Sigma_{p}=10 \mathrm{~S}$, Hall height integrated conductivity, $\Sigma_{h}=20 \mathrm{~S}$ and ULF wave frequency, $\mathrm{f}=20$ $\mathrm{mHz}$. The electrostatic value from (1) for AA in this case is $A A_{\text {static }}=-0.85$. 
$|A A|$

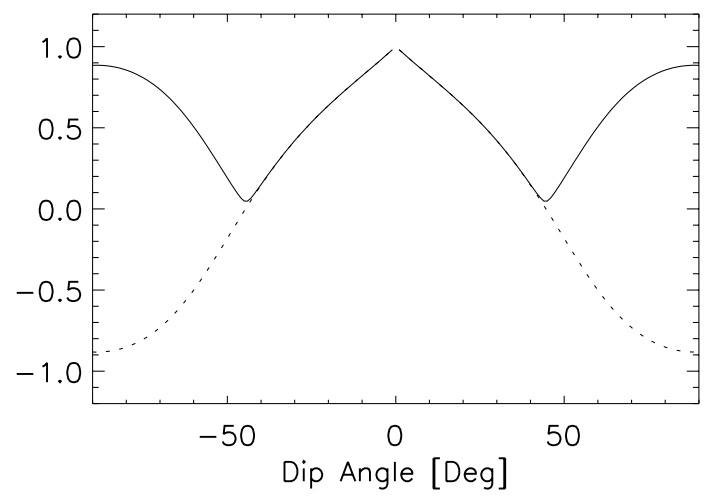

$|A F|$

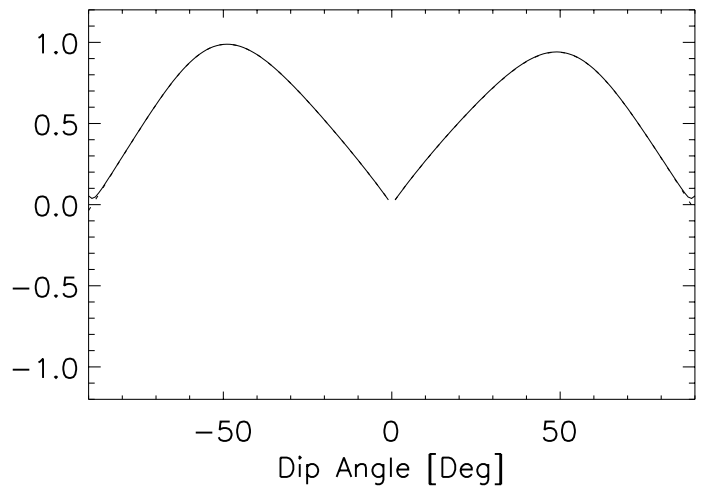

$|F A|$

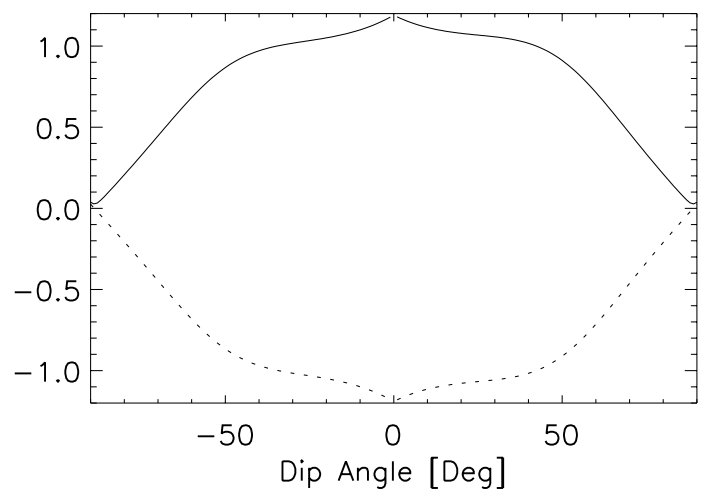

$|F F|$

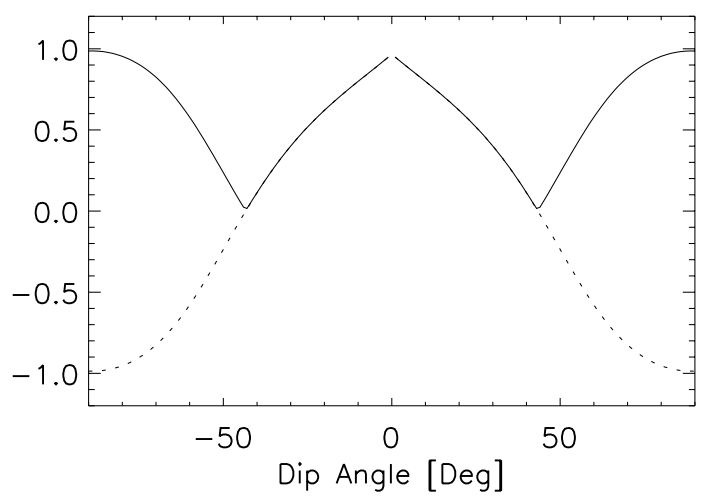

AA Phase
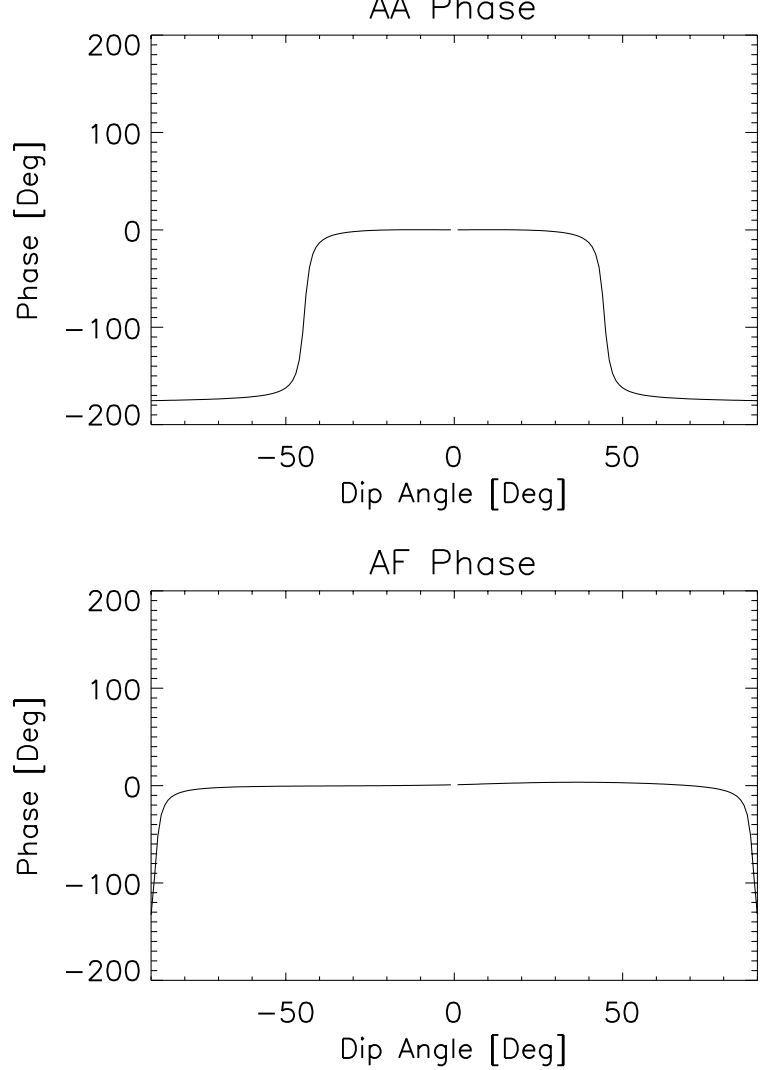

FA Phase

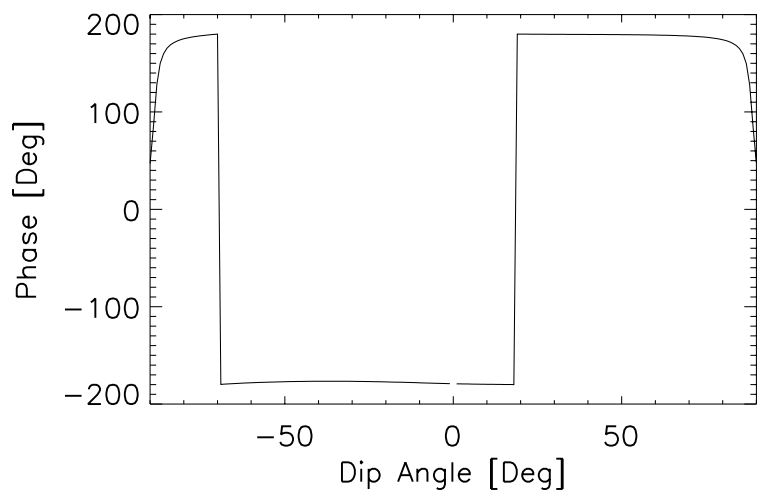

FF Phase

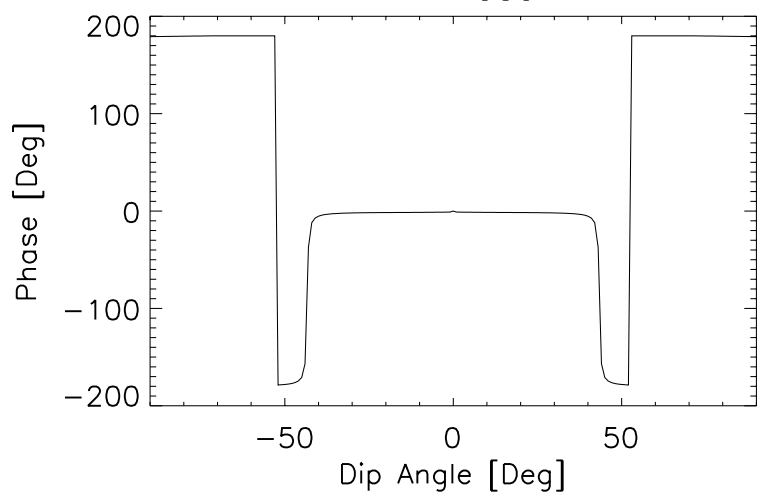

Figure 4. Reflection coefficient matrix elements for $k_{x}=k_{y}=\frac{\omega}{2 V_{a}}$, ionosphere height, $\mathrm{d}=1.25 \times 10^{5} \mathrm{~m}$, Alfvén speed, $V_{a}=2 \pi d$, direct height integrated conductivity, $\Sigma_{d}=1,000,000 \mathrm{~S}$, Pedersen height integrated conductivity, $\Sigma_{p}=10 \mathrm{~S}$, Hall height integrated conductivity, $\Sigma_{h}=20 \mathrm{~S}$ and ULF wave frequency, $\mathrm{f}=20 \mathrm{mHz}$. The electrostatic value from (1) for AA in this case is $A A_{\text {static }}=-0.85$. 

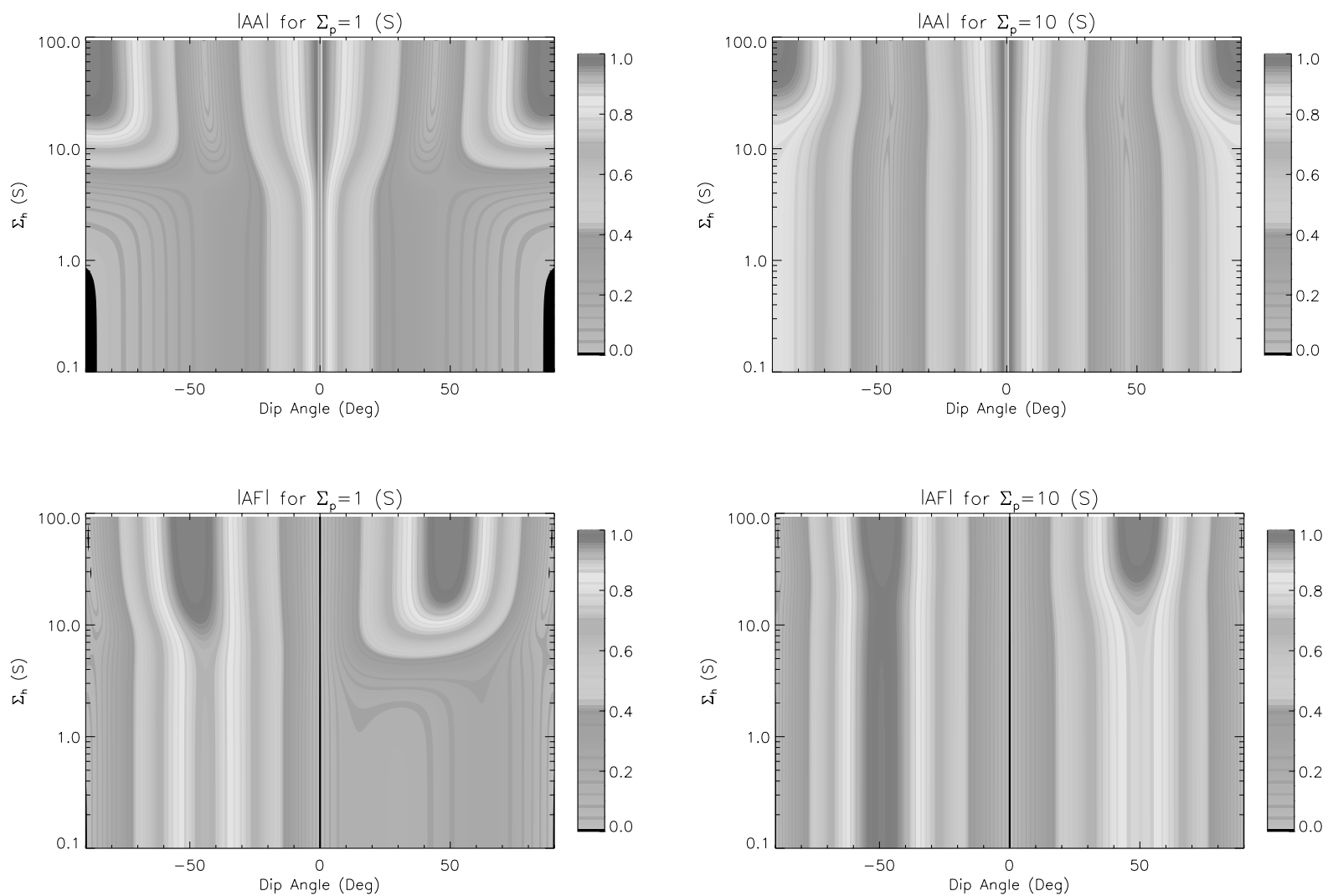

IFAl for $\Sigma_{\rho}=1(\mathrm{~S})$
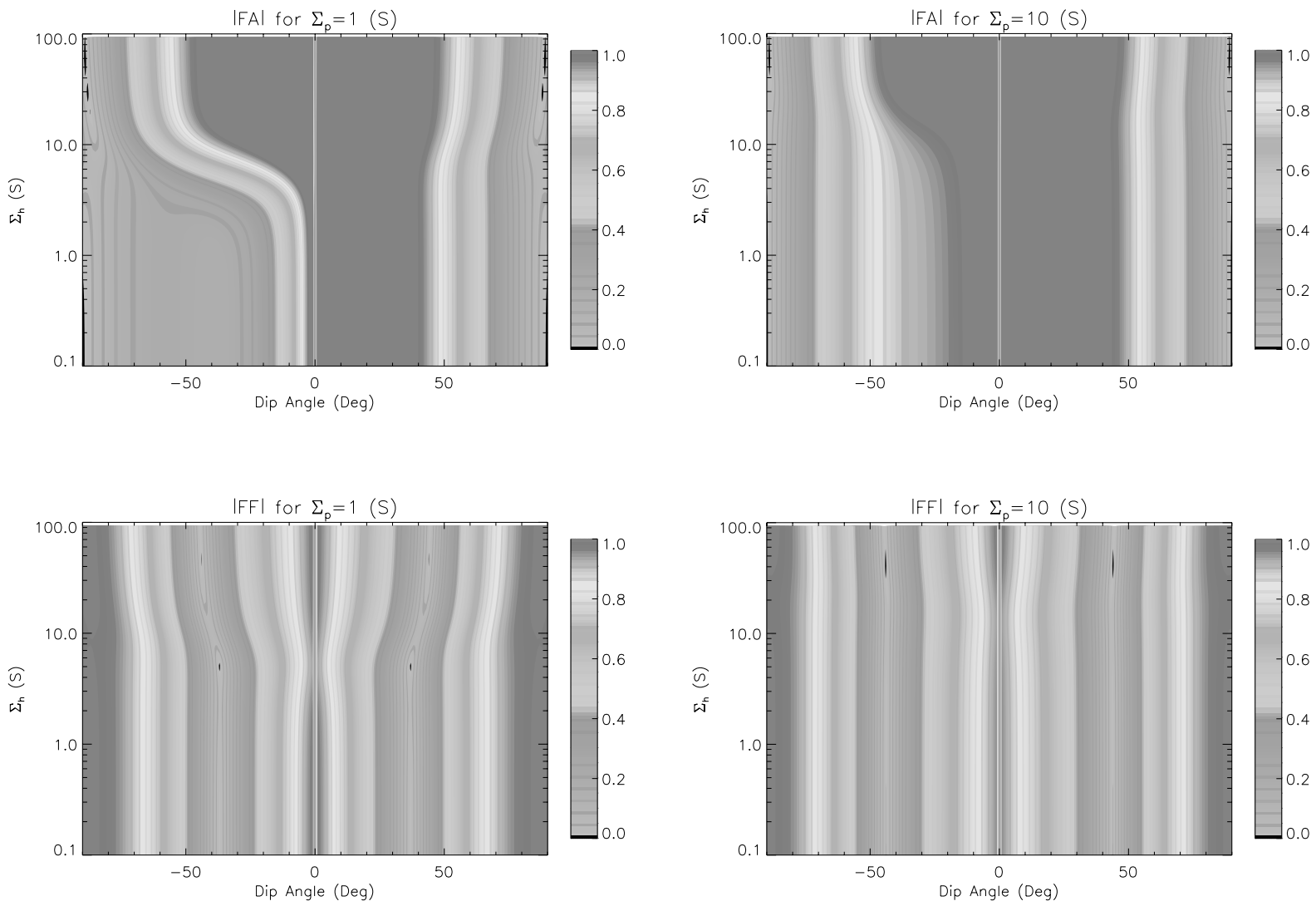

Figure 5. Reflection coefficient matrix elements for $k_{x}=k_{y}=\frac{\omega}{2 V}$, ULF wave frequency, $\mathrm{f}=20$ $\mathrm{mHz}$, Alfvén speed, $V_{a}=2 \pi d, \Sigma_{d}=1,000,000 \mathrm{~S}, \Sigma_{p}=1 \mathrm{~S}$ (left-hand panels) and $\Sigma_{p}=10 \mathrm{~S}$ (righthand panels), $\Sigma_{h}=\alpha$ for $0.1<\alpha<100 \mathrm{~S}$. 

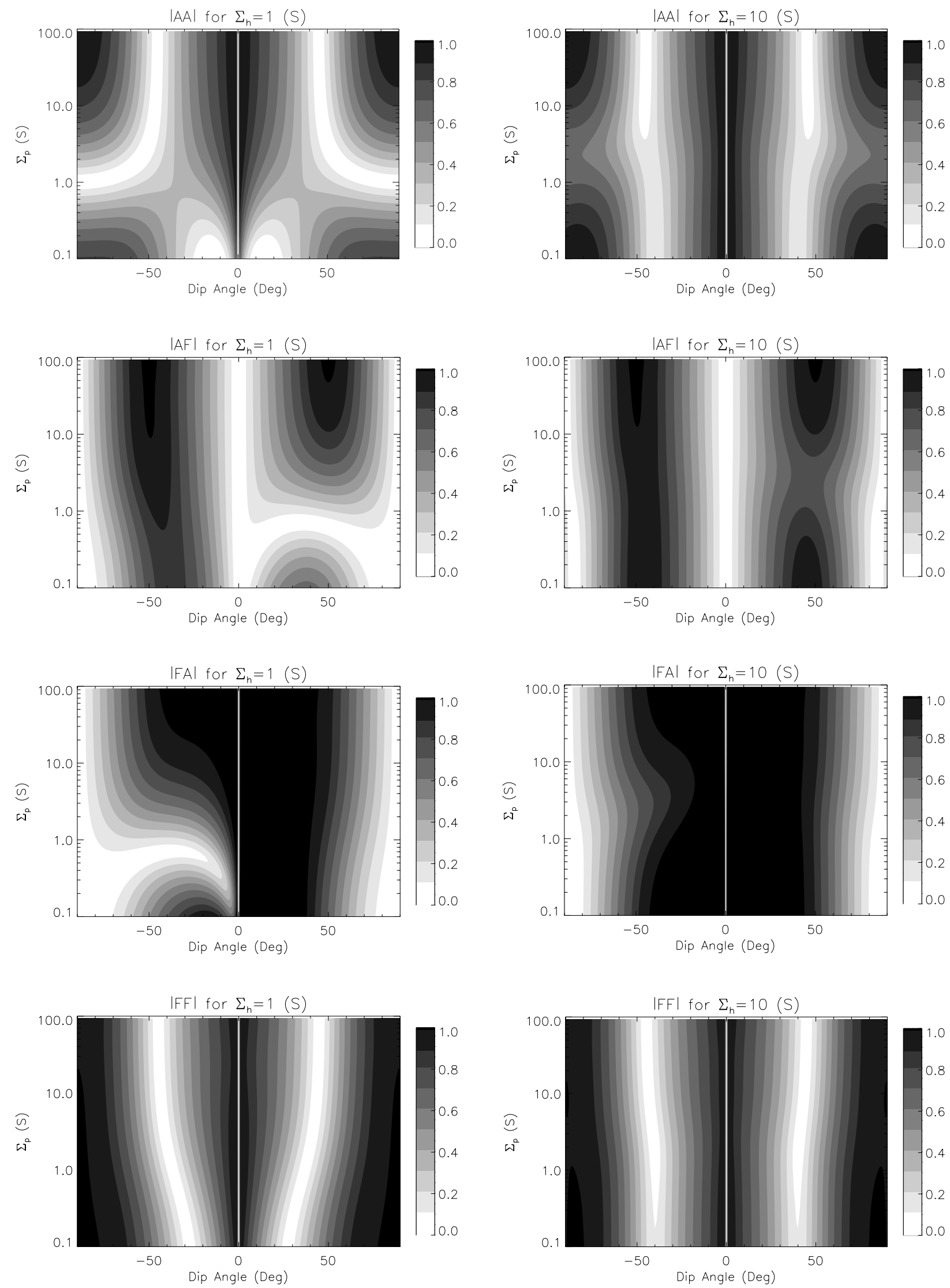

Figure 6. Reflection coefficient matrix elements for $k_{x}=k_{y}=\frac{\omega}{2 V}$, ULF wave frequency, $\mathrm{f}=20$ $\mathrm{mHz}$, Alfvén speed, $V_{a}=2 \pi d, \Sigma_{d}=1,000,000 \mathrm{~S}, \Sigma_{h}=1 \mathrm{~S}$ (left-hand panels) and $\Sigma_{h}=10 \mathrm{~S}$ (right-hand panels), $\Sigma_{p}=\alpha$ for $0.1<\alpha<100 \mathrm{~S}$. 
the dependence of wave mode conversion on conductivity and hemisphere.

\section{Discussion}

[31] The relationship between the $\vec{B}_{0}$ dip angle, ionosphere conductivity and ULF wave reflection and conversion for various horizontal wave structures is quite complex. The actual expressions for the various influences and their importance are all specified in the elements of the RCM given by (37)-(40), complicated by multiplication of the inverse matrix as specified in (45). Simplified analytic expressions for each coefficient may be obtained for vertical and horizontal magnetic fields and/or zero wave numbers. When all these parameters are non zero, the analytic expressions become very complicated and we resort to numeric solutions of (45). The general trends and relationships between the various parameters can be deduced from two dimensional plots such as Figures 5 and 6 . In this section, we discuss ULF wave reflection and conversion in the presence of oblique $\vec{B}_{0}$ by considering the wave electric fields in the various media.

[32] Assume that a unit amplitude, shear Alfvén or fast mode ULF wave is incident from the MHD region. The wave electric fields in the atmosphere, ionosphere and magnetosphere may be found using (24) and (45). The boundary conditions specify that the horizontal electric fields across the ionosphere boundary are continuous. However, the vertical component of the electric field in each region may be different. The difference between the vertical electric field in the atmosphere and magnetosphere indicates a charge density in the ionosphere. These parameters are shown in Figure 7 for the case where $k_{x}=k_{y}=\frac{\omega}{2 V_{a}}$ and for $\Sigma_{p}=10 \mathrm{~S}$ with $\Sigma_{h}$ varying from 0.1 to $100 \mathrm{~S}$. The RCM elements for these parameters are shown in the right-hand panels of Figure 5. There are regions where the vertical electric fields in the magnetosphere differ from those in the atmosphere with the associated charge separation in the ionosphere. The asymmetry around $I=0$ depends on the value of $k_{x}$. For the case where the incident ULF energy is a mix of the two modes, the variation of vertical electric fields and charge with magnetic field dip angle would be a proportional mix of these plots. For both incident ULF wave modes, the vertical electric fields diminish when $\Sigma_{h}$ is approximately equivalent to $\Sigma_{p}$.

[33] For a vertical $\vec{B}_{0}$, the vertical electric field is zero in the magnetosphere while the free charge at the boundary is large due to a vertical electric field in the atmosphere (Figure 7). If there is no shear Alfvén mode in the magnetosphere then $\nabla \cdot \vec{e}=0$ in both the magnetosphere and atmosphere. Since $k_{x}, k_{y}, e_{x}$ and $e_{y}$ are continuous across the boundary, then $e_{z}=0$ in the atmosphere with no difference in the vertical electric fields across the boundary as seen for the incident fast mode case. Therefore, for vertical $\vec{B}_{0}$, the magnitude of the charge depends on the presence of the shear Alfvén mode where $\nabla \cdot \vec{e} \neq 0$. The magnitude of the difference in $e_{z}$ is larger near vertical $\vec{B}_{0}$ for incident shear Alfvén waves while for the fast mode, the difference in $e_{z}$ is larger for midlatitudes. For a given frequency, conductivity and wave number, the variation of the dip angle is sufficient to alter the reflection and mode conversion properties so that for oblique $\vec{B}_{0}$ the charge at the ionosphere boundary also depends on a non zero $e_{z}$ in the magnetosphere.

[34] In a simplified model, there are a number of approximations that are necessary so that the problem may be solved. For the analytic treatment of ULF wave propagation through a thin sheet ionosphere in the presence of oblique magnetic fields, the following simplifications have been included:

1. The vertical current in the thin sheet ionosphere is zero. We expect this approximation to be more serious for dip angles near zero. ULF wave propagation for equatorial magnetic fields has been treated by Zhang and Cole [1995] and Waters et al. [2001].

2. The incident and reflected ULF wave polarization is linear. The more general case for circular or elliptical polarization of the incident wave requires complex horizontal wave numbers. The mathematical development in this paper allows for complex wave numbers.

3. Both ULF wave modes propagate in the MHD medium. In reality, the fast mode may become evanescent particularly where the Alfvén speed increases in the upper ionosphere. Nonpropagating modes $\left(k_{x}, k_{y}>\frac{\omega}{V_{s}}\right)$ are easily treated for vertical dip angles as shown by Yoshikawa and Itonaga [1996].

4. The atmosphere only contains evanescent, TEM wave modes. This is a reasonable approximation for frequencies $<$ $100 \mathrm{mHz}$. However, for higher frequencies (e.g., Pc 1), propagating TE and/or TM wave modes may exist with the additional possibility of an ionospheric waveguide [e.g., Manchester, 1968].

5 . The ionosphere conductivity is horizontally uniform and the horizontal wave numbers are constant throughout the model. Uniform horizontal conductivity is a standard approximation in these types of problems [e.g., Hughes, 1974]. The introduction of non uniform horizontal conductivity alters the wave polarization properties by introducing additional electric fields at the conductivity discontinuities [e.g., Glassmeier, 1984]. Keeping the horizontal wave numbers constant across the thin sheet may be justified, in the uniform conductivity case, from arguments based on Snell's law.

[35] The RCM elements show changes around a dip angle of $60^{\circ}$. This is particularly noticeable for $k_{x}=0$ and $k_{x}=k_{y}=\frac{\omega}{2 V_{a}}$ (Figures 3 and 4). This feature depends on the magnitude of $k_{y}$ and involves the multiplier of $\frac{\omega}{V_{a}}$. The dispersion relations, RCM elements and polarization vectors all contain $\cos (I)$ and $\sin (I)$. If we set $\cos (I)$ equal to the multiplier, which was 0.5 , then $I=60^{\circ}$.

\section{Conclusion}

[36] Wave properties from the interaction of ULF waves with the magnetosphere-ionosphere-atmosphere system in the presence of oblique background magnetic fields have been investigated. The reflection and mode conversion characteristics are defined by the RCM which contains complicated expressions that depend on wave frequency and wave numbers, ionosphere conductivity and magnetic field dip angles. The value of the wave number perpendicular to the plane that contains the background magnetic field $\left(k_{y}\right)$ has a major effect on 
a
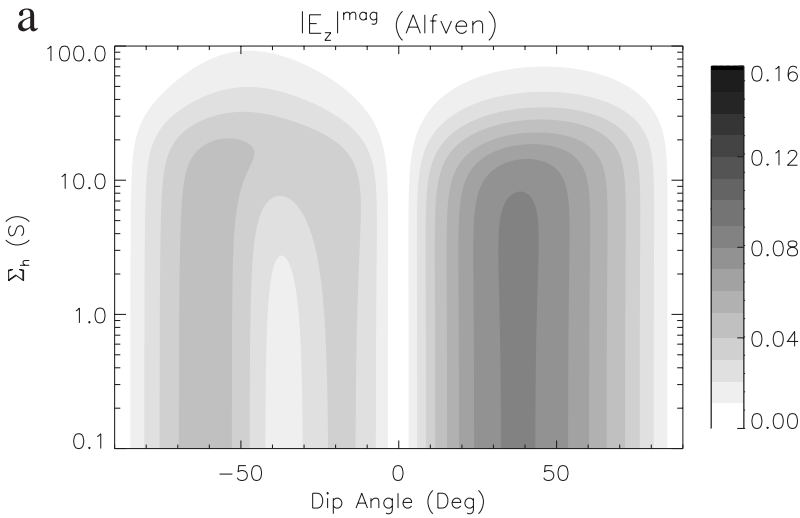

$\mathrm{b}$
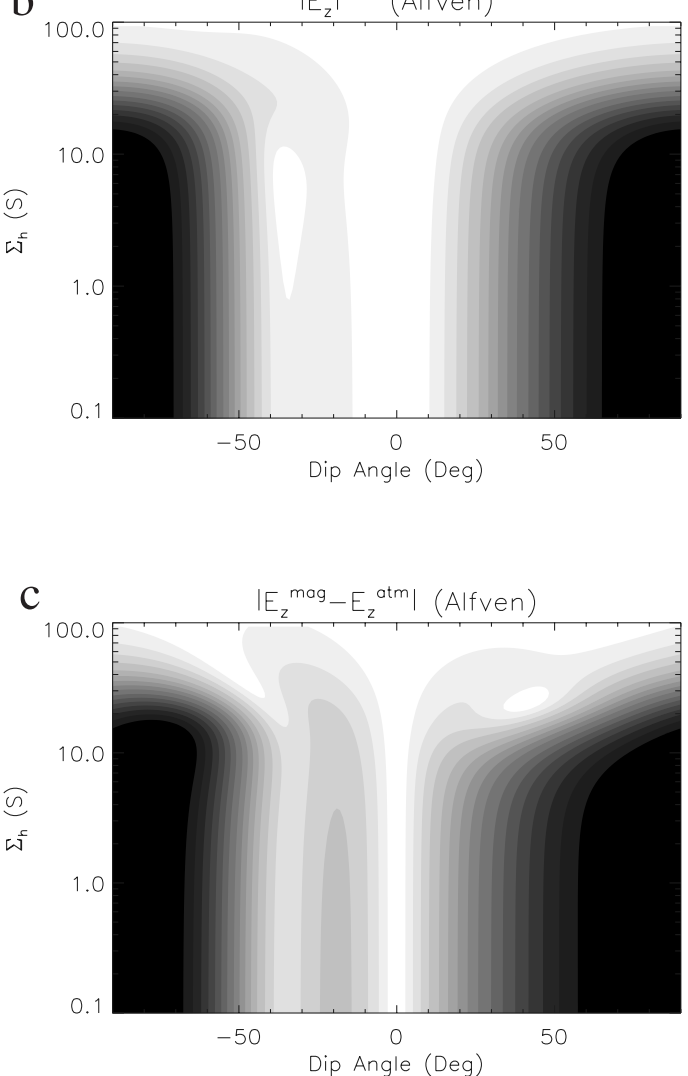
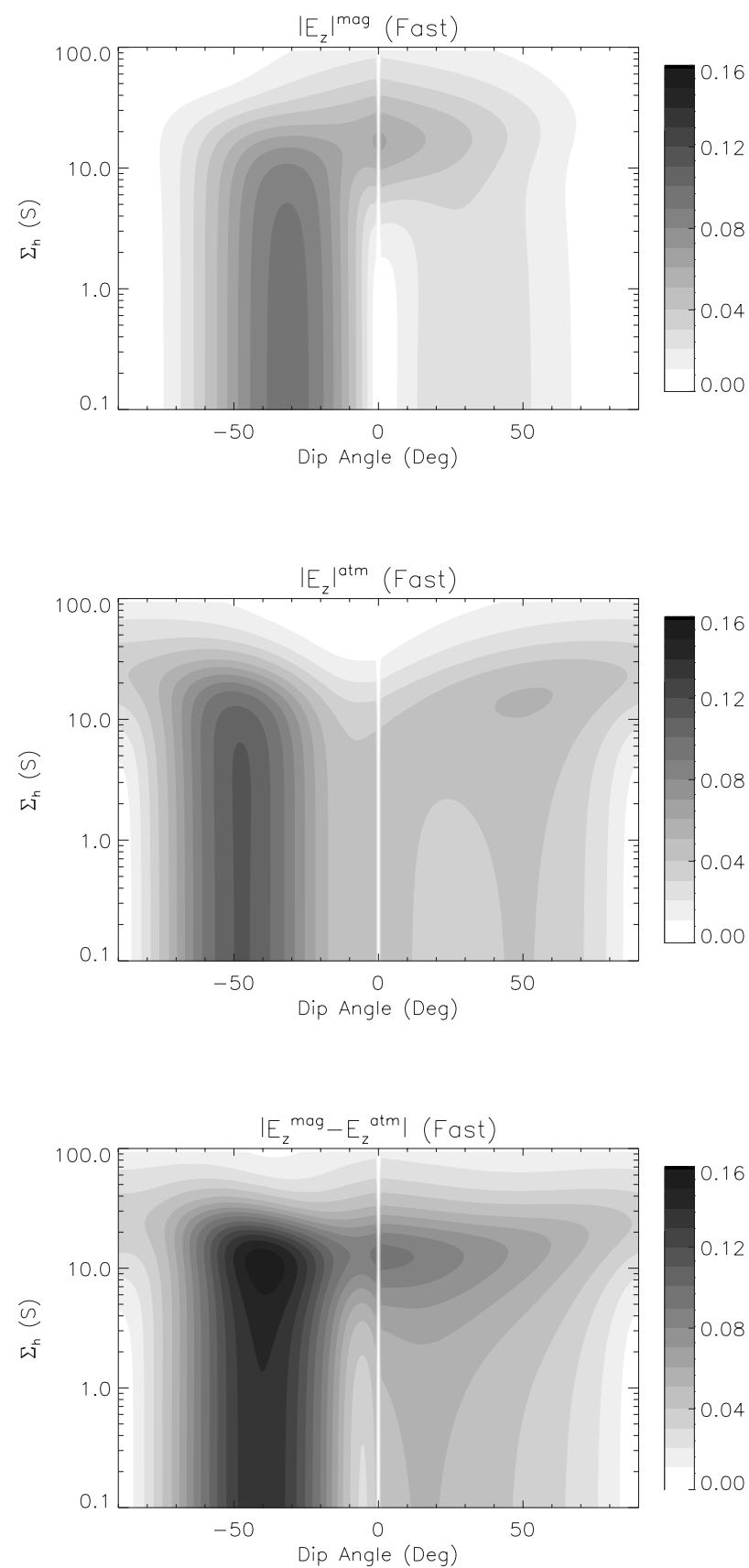

Figure 7. (a, b) Magnetospheric and atmospheric total vertical electric fields, $e_{z}$, for a unit incident shear Alfvén and fast mode where $k_{x}=k_{y}=\frac{\omega}{2 V_{a}}, V_{a}=2 \pi d, \Sigma_{d}=1,000,000 \mathrm{~S}, \Sigma_{p}=10 \mathrm{~S}, \Sigma_{h}=\alpha$ for $0.1<$ $\alpha<100$ and $\mathrm{f}=20 \mathrm{mHz}$. (c) Difference in magnetospheric and atmospheric $e_{z}$ for incident shear Alfvén and fast mode for the parameters in $(a, b)$.

the RCM. The results show that reflection properties for vertical fields (high-latitude regions) can greatly differ from those at lower latitudes, due solely to the background magnetic field dip angle. These are highly dependent on the spatial structure of ULF wave energy and experimental estimates of these parameters are required. Further work is focusing on a more realistic vertical ionosphere profile and numerical solutions. The assumption of ideal MHD conditions at the top boundary of the model is also under consideration.
[37] Acknowledgments. The authors would like the thank A.D.M. Walker, A. Karol and W.P. Wood for helpful discussions. This work was supported by grants from the Australian Research Council, the University of Newcastle and the Cooperative Research Center for Satellite Systems.

[38] Janet G. Luhmann thanks both of the referees for their assistance in evaluating this paper.

\section{References}

Alfvén, H., and C. G. Fälthammar, Cosmical Electrodynamics, Oxford Univ. Press, New York, 1963 
Alperovich, L. S., and E. N. Fedorov, On hydromagnetic wave beams propagation through the ionosphere, Ann. Geophys., 10, 647, 1992.

Altman, C., and H. Cory, The generalized thin-film optical method in electromagnetic wave propagation, Radio Sci., 4, 459, 1969.

Budden, K. G., The Propagation of Radio Waves. Cambridge Univ. Press, New York, 1985.

Cheng, D., Field and Wave Electromagnetics, Addison-Wesley-Longman, Reading, Mass., 1983.

Cross, R., An Introduction to Alfvén Waves, IOP, Bristol, U.K., 1988.

Dungey, J. W., The structure of the exosphere, or adventures in velocity space, in Geophysics: The Earth's Environment, edited by C. De Witt, J. Hieblot, and A. Lebeau, Gordon and Breach, Newark, N. J., 1963.

Ellis, P., and D. J. Southwood, Reflection of Alfvén waves by non-uniform ionospheres, Planet. Space Sci., 31, 107, 1983.

Fraser, B. J., Ionospheric duct propagation and Pc 1 pulsation sources, J. Geophys. Res., 80, 2790, 1975.

Glassmeier, K. H., On the influence of ionospheres with non-uniform conductivity distribution on hydromagnetic waves, J. Geophys., 54, 125, 1984.

Hughes, W. J., The effect of the atmosphere and ionosphere on long period magnetospheric micropulsations, Planet. Space Sci., 22, 1157, 1974.

Hughes, W. J., and D. J. Southwood, The screening of micropulsation signals by the atmosphere and ionosphere, J. Geophys. Res., 81, 3234, 1976 a.

Hughes, W. J., and D. J. Southwood, An illustration of modification of geomagnetic pulsation structure by the ionosphere, J. Geophys. Res., 81, 3241, 1976b.

Manchester, R. N., Correlation of Pc 1 micropulsations at spaced stations, J. Geophys. Res., 73, 3549, 1968.

Menk, F. W., B. J. Fraser, C. L. Waters, C. W. S. Ziesolleck, Q. Feng, S. H. Lee, and P. W. McNabb, Ground measurements of low latitude magnetospheric field line resonances, in Solar Wind Sources of Magnetospheric Ultra Low Frequency Waves, Geophys. Monogr. Ser., vol. 81, edited by M. J. Engebretson, K. Takahashi, and M. Scholer, p. 299, AGU, Washington, D.C., 1994.

Menk, F. W., R. A. Marshall, P. W. McNabb, and I. S. Dunlop, An experiment to study the effects of geomagnetic fluctuations on ionospheric HF, radio paths, J. Elect. Electron. Eng. Aust., 1995.

Nishida, A., Ionospheric screening effect and storm sudden commencement, J. Geophys. Res., 69, 1861, 1964.

Nygren, T., A simple method for obtaining reflection a transmission coefficients and fields for an electromagnetic wave in a horizontally stratified ionosphere, Planet. Space Sci., 29, 521, 1981.

Nygren, T., A method of full wave analysis with improved stability, Planet. Space Sci., 30, 427, 1982.

Pitteway, M. L. V., The numerical calculation of wave fields, reflection coefficients and polarizations for long radio waves in the lower ionosphere, Philos. Trans. R. Soc. London, Ser, 257, 243, 1965.
Poole, A. W. V., P. R. Sutcliffe, and A. D. M. Walker, The relationship between ULF geomagnetic pulsations and ionospheric doppler oscillations: Derivation of a model, J. Geophys. Res., 93, 14,656, 1988.

Priest, E. R., Solar Magnetohydrodynamics, D. Reidel, Norwell, Mass., 1982.

Prince, C. E., and F. X. Bostick, Ionospheric transmission of transversely propagated plane waves at micropulsation frequencies and theoretical power spectrums, J. Geophys. Res., 69, 3213, 1964.

Scholer, M., On the motion of artifical ion clouds in the magnetosphere, Planet. Space Sci., 18, 977, 1970.

Sorokin, V. M., The role of the ionosphere in the propagation of geomagnetic pulsations, Geomagn. Aeron., 26, 532, 1986.

Stix, T. H., The Theory of Plasma Waves, McGraw-Hill, New York, 1962.

Tamao, T., Direct contribution of oblique field-aligned currents to the ground magnetic fields, J. Geophys. Res., 91, 183, 1986.

Ullah, N., and S. L. Kahalas, Coupling of magnetohydrodynamic to electromagnetic waves at a plasma discontinuity, 1, Radiation field, Phys. Fluids, 6, 284, 1963.

Waters, C. L., F. W. Menk, and B. J. Fraser, The resonant structure of low latitude Pc 3 geomagnetic pulsations, Geophys. Res. Lett., 18, 2293, 1991.

Waters, C. L., F. W. Menk, and B. J. Fraser, Low latitude geomagnetic field line resonances: Experiment and modeling, J. Geophys. Res., 99, 17,547, 1994.

Waters, C. L., M. D. Sciffer, B. J. Fraser, K. Brand, K. Foulkes, F. W. Menk, O. Saka, and K. Yumoto, The phase structure of very low latitude ULF waves across dawn, J. Geophys. Res., 106, 15,599, 2001.

Yoshikawa, A., and M. Itonaga, Reflection of shear Alfven waves at the ionosphere and the divergent Hall current, Geophys. Res. Lett., 23, 101, 1996.

Yoshikawa, A., and M. Itonaga, The nature of reflection and mode conversion of MHD waves in the inductive ionosphere: Multistep mode conversion between divergent and rotational electric fields, J. Geophys. Res., $105,10,565,2000$.

Yoshikawa, A., M. Itonaga, S. Fujita, H. Nakata, and K. Yumoto, Eigenmode analysis of field line oscillations interacting with the ionosphere-atmosphere-solid earth electromagnetic coupled system, J. Geophys. Res., 104, 28,437, 1999.

Zhang, D. Y., and K. D. Cole, Formulation and computation of hydromagnetic wave penetration into the equatorial ionosphere and atmosphere, J. Atmos. Terr. Phys., 57, 813, 1995.

M. D. Sciffer and C. L. Waters, School of Mathematical and Physical Sciences, University of Newcastle, Callaghan 2308, New South Wales, Australia. (physpuls8@cc.newcastle.edu.au) 